\title{
Dosimétrie du radon : les points de vue de l'UNSCEAR et de la CIPR sont-ils contradictoires?
}

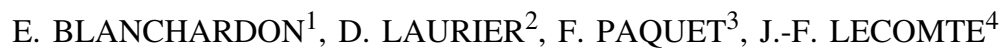 \\ et M. TIRMARCHE ${ }^{4}$
}

(Manuscrit reçu le 6 janvier 2009, accepté le 17 mars 2009)

RÉSUMÉ La conversion en dose d'une exposition au radon fait l'objet de débats. L'UNSCEAR a défini un coefficient de conversion de la concentration en radon vers la dose efficace à partir de modèles dosimétriques et d'informations épidémiologiques. La CIPR recommande d'évaluer le risque lié au radon à partir des études épidémiologiques. Pour permettre la prise en compte d'une exposition combinée à d'autres sources de rayonnements ionisants, elle a adopté des conventions de conversion en dose efficace inférieures de 10 à $30 \%$ au coefficient de l'UNSCEAR, sur la base du suivi épidémiologique des mineurs d'uranium. Ainsi, des niveaux de référence différents pour la concentration en radon peuvent être déduits d'un même niveau de dose efficace suivant que l'on applique l'un ou l'autre facteur de conversion. Cependant, il convient de rappeler que ces différences sont négligeables par rapport aux incertitudes associées aux études tant dosimétriques qu'épidémiologiques et que les niveaux de référence ne sont que des outils pour aider à réduire aussi bas que raisonnablement possible l'exposition au radon et le risque qui en résulte.

ABSTRACT Radon dosimetry: Is there a contradiction between UNSCEAR and ICRP positions? The conversion of radon exposure into dose is being debated. The UNSCEAR defined a coefficient for conversion of radon concentration into effective dose from dosimetric models and epidemiological studies. The ICRP recommends evaluating the risk associated with radon exposure from epidemiological studies. To account for possible combined exposure to other sources of ionizing radiation, the ICRP adopted, on the basis of the epidemiological follow-up of uranium miners, effective dose conversion conventions which are 10 to $30 \%$ lower than the UNSCEAR coefficient. As a consequence, different reference levels for radon concentration can be derived from a single level of effective dose by applying either conversion factor. Still, it should be stressed that these differences are negligible when compared with the uncertainty associated with both dosimetric and epidemiological studies, and that the reference levels are mainly tools to help in reducing radon exposure and the associated risk as low as reasonably achievable.

Keywords: radon / radon progeny / dose / effective dose / dosimetry / dose coefficient / conversion convention / UNSCEAR / ICRP

\footnotetext{
IRSN, Laboratoire d'évaluation de la dose interne, DRPH/SDI/LEDI, BP 17, 92262 Fontenay-aux-Roses Cedex, France. IRSN, Laboratoire d'épidémiologie, DRPH/SRBE/LEPID, BP 17, 92262 Fontenay-aux-Roses Cedex, France. IRSN, Direction scientifique, BP 3, 13115 Saint Paul Lez Durance Cedex, France.

4 IRSN, Division des relations internationales, DSDRE/DRI, BP 17, 92262 Fontenay-aux-Roses Cedex, France.
} 


\section{Facteurs de conversion en dose}

Les études épidémiologiques conduites sur des populations de mineurs ont démontré l'existence d'une association entre le risque de cancer du poumon et l'exposition cumulée au radon (NRC, 1999). En conséquence, le radon a été classé comme cancérogène pulmonaire certain pour l'homme par le Centre international de recherche sur le cancer en 1988 (CIRC, 1988). À l'heure actuelle, le cancer du poumon apparait comme la seule pathologie présentant une association avérée avec l'exposition au radon (UNSCEAR, 2008).

La conversion en dose d'une exposition au radon fait cependant l'objet de débats au sein de la communauté de la radioprotection. Les points de vue respectifs du comité scientifique des nations unies sur les effets des radiations (UNSCEAR) et de la commission internationale de protection radiologique (CIPR) ont été analysés et sont présentés dans cette note. Bien qu'empruntant des approches différentes, basées respectivement sur la dosimétrie et sur l'épidémiologie, l'UNSCEAR et la CIPR ont défini des facteurs de conversion en dose très similaires dont les valeurs sont reproduites dans le tableau I.

L'essentiel de la dose reçue par le poumon ne provient pas du gaz radon luimême mais des descendants à vie courte avec lesquels il est en équilibre partiel. La concentration équivalente en radon à l'équilibre (EEC) d'un mélange de descendants du radon est la concentration en activité de radon en équilibre radioactif avec ses descendants à vie courte ayant la même concentration d'énergie potentielle alpha. Le facteur d'équilibre $(F)$ est le rapport entre l'EEC et l'activité volumique du gaz radon.

L'unité historique de mesure de l'exposition au radon dans les mines est le working level month (WLM) qui correspond à une exposition à une concentration ambiante en énergie potentielle alpha des descendants à vie courte du radon de $1,3 \times 10^{8} \mathrm{MeV} \mathrm{m}^{-3}$ pendant $170 \mathrm{~h}$. Soit

$$
\begin{gathered}
1 \mathrm{WLM}=3,54 \times 10^{-3} \mathrm{~J} \mathrm{~h} \mathrm{~m}^{-3}, \\
1 \mathrm{WLM}<=>6,37 \times 10^{5} \mathrm{~Bq} \mathrm{~h} \mathrm{~m}^{-3} \mathrm{EEC}, \\
1 \mathrm{WLM}<=>6,37 \times 10^{5} / F \mathrm{~Bq} \mathrm{~h} \mathrm{~m}^{-3} \text { de gaz radon. }
\end{gathered}
$$

Lorsque le facteur d'équilibre est fixé à une valeur $F=0,4$ typique d'un intérieur naturellement ventilé, et pour des temps de présence de $2000 \mathrm{~h} / \mathrm{an}$ au travail et 
TABLEAU I

Correspondance entre l'exposition au radon et la dose efficace suivant l'UNSCEAR (2000, 2008) et la CIPR $(1993,2007)$. Les valeurs suivantes sont attribuées aux paramètres des modèles : facteur de pondération pour le rayonnement alpha $w_{R}=20$; facteur de pondération tissulaire pour les poumons $w_{T}=0,12$; fraction libre de l'énergie potentielle alpha des descendants du radon $f_{p}=0,025$; diamètre médian en activité des particules auxquelles sont attachés les descendants du radon $A M D=0,2 \mu \mathrm{m}$; débit respiratoire $B=0,8 \mathrm{~m}^{3} \mathrm{~h}^{-1}$. Les valeurs numériques sont arrondies à un seul chiffre significatif.

Correspondence between radon exposure and effective dose according to UNSCEAR (2000, 2008) and ICRP (1993, 2007). The parameters of the models are set at the following values: radiation weighting factor for alpha particles $w_{R}=20$; tissue weighting factor for lung $w_{T}=0.12$; unattached fraction of potential alpha energy from radon progeny $f_{p}=0.025$; activity median diameter of the particles which the radon progeny is attached to $\mathrm{AMD}=0.2 \mu \mathrm{m}$; breathing rate $B=0.8 \mathrm{~m}^{3} \mathrm{~h}^{-1}$. Numerical values are rounded to a single digit.

\begin{tabular}{|c|c|c|c|c|c|}
\hline \multicolumn{3}{|c|}{ Exposition } & \multicolumn{3}{|c|}{ Dose efficace } \\
\hline \multirow{2}{*}{\multicolumn{3}{|c|}{ exposition unitaire }} & UNSCEAR & & \\
\hline & & & population générale & public & travailleurs \\
\hline \multicolumn{3}{|c|}{$1 \mathrm{~Bq} \mathrm{~h} \mathrm{~m}{ }^{-3} \mathrm{EEC}$ de descendants du radon } & $9 \mathrm{nSv}$ & $6 \mathrm{nSv}^{*}$ & $8 \mathrm{nSv} *$ \\
\hline \multicolumn{3}{|c|}{$1 \mathrm{WLM}$} & $6 \mathrm{mSv}^{*}$ & $4 \mathrm{mSv}$ & $5 \mathrm{mSv}$ \\
\hline \multicolumn{6}{|c|}{ concentration en radon pendant 1 an } \\
\hline \multirow{2}{*}{$\begin{array}{c}\text { UNSCEAR } \\
\text { domicile }\end{array}$} & \multicolumn{2}{|c|}{ CIPR } & & & \\
\hline & domicile & lieu de travail & & & \\
\hline $40 \mathrm{~Bq} \mathrm{~m}^{-3}$ & $60 \mathrm{~Bq} \mathrm{~m}^{-3 *}$ & $150 \mathrm{~Bq} \mathrm{~m}^{-3 *}$ & & $1 \mathrm{mSv}$ & \\
\hline $400 \mathrm{~Bq} \mathrm{~m}^{-3} *$ & $600 \mathrm{~Bq} \mathrm{~m}^{-3}$ & $1500 \mathrm{~Bq} \mathrm{~m}^{-3}$ & & $10 \mathrm{mSv}$ & \\
\hline
\end{tabular}

* Valeur déduite par proportionnalité des valeurs publiées.

7000 h/an à domicile, on obtient les équivalences suivantes :

$1 \mathrm{~Bq} \mathrm{~m}^{-3}$ de radon durant 1 an $<=>4,4 \times 10^{-3}$ WLM à domicile,

$1 \mathrm{~Bq} \mathrm{~m}^{-3}$ de radon durant 1 an $<=>1,26 \times 10^{-3} \mathrm{WLM}$ au travail,

ainsi que les coefficients de conversion en dose du tableau I.

Lorsque la valeur de $F$ diffère de 0,4 la correspondance entre l'exposition exprimée en termes de WLM et la dose efficace n'est pas significativement modifiée. En revanche, la correspondance entre l'exposition à une concentration de gaz radon et la dose efficace indiquée dans le tableau I doit être multipliée par F/0,4.

\section{Point de vue de l'UNSCEAR}

Un modèle dosimétrique représente le dépôt des descendants à vie courte du radon, sous forme de particules nanométriques (fraction libre) ou attachés aux poussières atmosphériques, dans les voies respiratoires suivant leurs propriétés 
thermodynamiques et aérodynamiques, et l'irradiation qui s'ensuit. La contribution majeure à la dose résulte de la rapide décroissance par émission alpha des ${ }^{218} \mathrm{Po}$ et ${ }^{214} \mathrm{Po}$ avant leur épuration biologique du poumon. Le modèle dosimétrique doit donc rendre compte du transport des particules alpha sur quelques dizaines de microns depuis la lumière des voies respiratoires pour estimer le dépôt d'énergie dans les cellules radiosensibles suivant leur position supposée. La dose pulmonaire correspondante est pondérée d'un facteur $w_{R}$ pour tenir compte de l'efficacité biologique relative des particules alpha. Enfin, un facteur $w_{T}$, issu de l'épidémiologie et représentant la part de l'irradiation pulmonaire dans le détriment global, est appliqué pour calculer une dose efficace.

L'UNSCEAR a passé en revue plusieurs modèles dosimétriques dans ses publications de 1982 et 1988 (UNSCEAR, 1982, 1988), dont le modèle respiratoire de la publication 30 de la CIPR (ICRP, 1979). En conséquence, il a adopté un coefficient de conversion de la concentration en radon vers la dose efficace basé principalement sur les recommandations de l'agence à l'énergie nucléaire et de la CIPR (NEA, 1983; ICRP, 1981). D'autres modèles dosimétriques ont ensuite été pris en considération mais le coefficient de conversion n'a pas été modifié, à l'exception d'un changement mineur dans la valeur numérique du facteur d'équilibre utilisée dans le rapport de 1993 (UNSCEAR, 1993). Dans celui de 2000, il est indiqué que les modèles dosimétriques les plus récents, dont celui de la publication 66 de la CIPR (ICRP, 1994), suggèrent une augmentation du coefficient, mais aussi que les données épidémiologiques conduisent la CIPR à définir des facteurs de conversion plus faibles (UNSCEAR, 2000). En conséquence, le coefficient de conversion en dose du rapport UNSCEAR de 1988 a été jugé comme étant dans l'intervalle des valeurs calculées par les approches dosimétriques et épidémiologiques, et n'a pas été modifié. Ainsi, une exposition à $1 \mathrm{~Bq} \mathrm{~h} \mathrm{~m}^{-3}$ de radon à l'équilibre avec ses descendants à vie courte correspond à une dose efficace de $9 \mathrm{nSv}$; une exposition domestique de $40 \mathrm{~Bq} \mathrm{~m}^{-3}$ de radon conduit à une dose efficace annuelle de $1 \mathrm{mSv}$ (UNSCEAR, 2000).

Ce coefficient de dose s'applique à la population adulte en général. Pour extrapoler à un groupe d'âge inférieur à 10 ans, l'UNSCEAR suggère que le coefficient puisse être en moyenne plus élevé d'un facteur 1,5 à 2 que chez l'adulte (UNSCEAR, 1988). En dépit du niveau de précision apparent, l'UNSCEAR reconnait qu'une grande incertitude est associée à ce coefficient de dose. Dans son rapport de 1988, il estime que sa valeur peut varier d'un facteur 3 suivant la position des cellules cibles considérées dans la dosimétrie (UNSCEAR, 1988). En 1993, l'UNSCEAR souligne ce point et note que «l'incertitude prévaut à propos d'un coefficient de conversion adapté pour déduire la dose efficace de la concentration. » (UNSCEAR, 1993). 


\section{Point de vue de la CIPR}

L'approche de la CIPR est basée sur les études épidémiologiques des mineurs d'uranium, qui permettent une évaluation directe du risque de décès par cancer pulmonaire dû au radon (ICRP, 1993). La CIPR considère que cette approche introduit moins d'incertitude et est plus appropriée que l'application d'un modèle dosimétrique qui requerrait ensuite l'utilisation indirecte de l'épidémiologie des survivants des bombardements d'Hiroshima et de Nagasaki pour relier l'estimation d'une dose à celle d'un risque. Elle recommande donc que son modèle dosimétrique respiratoire humain (ICRP, 1994) ne soit pas utilisé pour l'évaluation et le contrôle des expositions au radon. Au lieu de cela, la CIPR estime le détriment sanitaire associé au radon à partir des études épidémiologiques des mineurs exposés au radon.

Sur cette base, la CIPR a retenu une probabilité vie entière de décès par cancer du poumon, ou coefficient de risque nominal, de $3 \times 10^{-4}$ par WLM pour l'exposition au radon des femmes et des hommes, travailleurs ou membres du public. Le coefficient de détriment, qui prend en compte l'occurrence d'un cancer mortel ou non ainsi que les années de vie perdues a été estimé similaire à cette valeur (ICRP, 1993).

Pour permettre la prise en compte de l'exposition combinée à d'autres sources de rayonnements ionisants, la CIPR propose néanmoins un facteur de conversion de l'exposition au radon en dose efficace. Il est obtenu par comparaison directe du détriment associé à une dose efficace unitaire ou à une exposition unitaire au radon. Dans les recommandations de 1990, le détriment par unité de dose efficace est de $5,6 \times 10^{-5}$ par $\mathrm{mSv}$ pour les travailleurs et $7,3 \times 10^{-5}$ par $\mathrm{mSv}$ pour le public (ICRP, 1991). En termes de détriment, une exposition au radon de 1 WLM est donc équivalente à une dose efficace de $5 \mathrm{mSv}$ pour les travailleurs et $4 \mathrm{mSv}$ pour le public (ICRP, 1993). La conversion ainsi obtenue est appelée convention de conversion. Elle est basée sur une égalité du détriment et non sur la dosimétrie.

La CIPR recommande également des niveaux d'action pour l'intervention. Ces niveaux sont fixés pour les habitations dans l'intervalle correspondant à des doses efficaces annuelles de 3 à $10 \mathrm{mSv}$ et en conséquence à des concentrations en radon comprises entre 200 et $600 \mathrm{~Bq} \mathrm{~m}^{-3}$. De façon similaire, des niveaux d'action sur les lieux de travail sont recommandés entre 500 et $1500 \mathrm{~Bq} \mathrm{~m}^{-3}$. La CIPR suggère néanmoins que les autorités nationales puissent utiliser des niveaux d'action différents en termes de concentration en radon, particulièrement pour les mines où le facteur d'équilibre diffère significativement de la valeur de référence utilisée (ICRP, 1993). 
Les recommandations 2007 de la CIPR soulignent que les études épidémiologiques récentes confirment un risque de cancer du poumon résultant de l'exposition au radon même à une concentration modérée. Les études du radon domestique (Darby et al., 2006 ; Krewski et al., 2006 ; Lubin et al., 2004) fournissent une méthode directe pour l'estimation des risques encourus dans l'habitat. Les niveaux d'action ont été remplacés par des niveaux de référence (niveaux plafonds et non plus planchers) à fixer par les autorités nationales en dessous de $10 \mathrm{mSv} / \mathrm{an}$, ce qui correspond à des concentrations en radon de $600 \mathrm{~Bq} \mathrm{~m}^{-3}$ pour les habitations et $1500 \mathrm{~Bq} \mathrm{~m}^{-3}$ pour les lieux de travail (ICRP, 2007).

\section{Changements en cours}

Les deux approches présentées ci-dessus évoluent avec l'avancement des connaissances scientifiques. Les modifications apportées par les différentes instances portent sur les coefficients de létalité ou de conversion en dose ou sur les niveaux de référence de la concentration en radon dans les habitations et sur les lieux de travail.

Dans son dernier rapport, l'UNSCEAR ne modifie pas son coefficient de dose pour l'inhalation de radon (UNSCEAR, 2008). Comme le montre le tableau I, celui-ci apparait légèrement différent de la convention de conversion en dose réutilisée par la CIPR dans ses dernières recommandations (ICRP, 2007). Cette différence entre facteurs de conversion pourrait passer inaperçue si elle n' avait des conséquences sur les niveaux en dessous desquels il est recommandé de ramener la concentration en radon. L'organisation mondiale de la santé (OMS) devrait prochainement recommander que les niveaux de référence nationaux soient choisis dans l'intervalle $100-400 \mathrm{~Bq} \mathrm{~m}^{-3}$ (WHO, 2007). L'agence internationale à l'énergie atomique (AEIA) pourrait adopter à la fois les niveaux de référence de la CIPR en termes de dose efficace et le coefficient de conversion en dose de l'UNSCEAR, ce qui impliquerait un niveau de référence de la concentration en radon au maximum de $400 \mathrm{~Bq} \mathrm{~m}^{-3}$ pour la population en général et $1000 \mathrm{~Bq} \mathrm{~m}^{-3}$ pour les lieux de travail.

La CIPR, qui recommande de fixer des valeurs de référence inférieures à 600 et $1500 \mathrm{~Bq} \mathrm{~m}^{-3}$ pour respectivement les habitations et les lieux de travail (ICRP, 2007), a formé un groupe de travail pour faire le point sur la connaissance des risques liés aux émetteurs alpha. Une partie importante du travail de ce groupe concerne l'exposition au radon et devrait permettre la remise à jour de la publication 65 de la CIPR (ICRP, 1993) par la prise en compte des résultats les plus récents des études épidémiologiques de l'exposition au radon dans l'habitat et dans les mines. 


\section{Discussion}

Malgré des disparités apparentes, il n'y a pas de véritable contradiction entre les recommandations en préparation par l'OMS et l'AEIA et celles publiées par la CIPR. L'OMS et l'AEIA pourraient proposer des niveaux de référence inférieurs à $400 \mathrm{~Bq} \mathrm{~m}^{-3}$ pour le public (respectivement inférieurs à $1000 \mathrm{~Bq} \mathrm{~m}^{-3}$ pour les lieux de travail). Si tel était le cas, ces niveaux seraient cohérents, quoique plus contraignants, avec la recommandation de la CIPR (ICRP, 2007) faite aux autorités nationales de fixer de tels niveaux de référence en dessous de $600 \mathrm{~Bq} \mathrm{~m}^{-3}$ (respectivement en dessous de $1500 \mathrm{~Bq} \mathrm{~m}^{-3}$ ).

Il demeure néanmoins une différence d'environ $10 \%$ pour les travailleurs et $30 \%$ pour les membres du public entre les facteurs de conversion de la concentration en radon vers la dose efficace proposés respectivement par l'UNSCEAR, à partir de modèles dosimétriques, et par la CIPR, à partir des études épidémiologiques des mineurs d'uranium.

Cette différence peut sembler importante, mais elle reste négligeable sur le plan scientifique au regard des incertitudes considérables associées aux approches dosimétrique et épidémiologique. Comme l'explique la publication 65 de la CIPR, « les sources d'incertitude dans l'épidémiologie du radon sont la limite statistique imposée par la taille de la population exposée, la nécessité de choisir un modèle de projection pour estimer les risques sur la vie entière et de postuler une relation dose-effet pour obtenir des estimations du risque à des niveaux d'exposition inférieurs à ceux pour lesquels il existe un excès de risque directement observable. En plus de ces incertitudes statistiques, il existe plusieurs sources d'incertitude non stochastique dans ces études : l'incertitude sur les estimations d'exposition individuelle, la difficulté à choisir un groupe témoin approprié, les différentes atmosphères de travail dans les mines, incluant l'influence d'autres poussières de minerai non radioactif, les différents niveaux de tabagisme et les différences (jusqu'à un facteur d'environ deux) dans les périodes moyennes de suivi. De plus l'énergie potentielle alpha inhalée pourrait ne pas être la quantité la plus pertinente pour mesurer l'exposition, en raison de la variabilité de paramètres physiques tels que la distribution en taille des particules composant les aérosols inhalés » (ICRP, 1993). Quantitativement, la CIPR (ICRP, 1993) rapporte un intervalle de confiance à $95 \%$ d'un facteur trois, entre 0,8 et 2,1 par WLM, sur l'excès de risque relatif déduit de la moyenne pondérée de sept études épidémiologiques de mineurs exposés au radon.

Parallèlement, les sources d'incertitude dans le calcul dosimétrique incluent la définition des cellules cibles pour l'initiation des tumeurs avec leurs positions et leurs radiosensibilités respectives, la pondération des contributions des différentes 
régions du poumon à la dose efficace, la valeur du facteur de pondération $w_{R}$ pour les rayonnements alpha, le débit respiratoire, la fraction attachée des descendants $\mathrm{du}$ radon, le facteur d'équilibre, la distribution des diamètres des particules auxquelles les descendants du radon sont attachés, le modèle retenu pour représenter le dépôt de l'aérosol dans le poumon, l'épuration muco-ciliaire et l'absorption vers le sang des descendants du radon. Les principales évaluations de dose absorbée par les cellules épithéliales du poumon recensées par l'UNSCEAR (2000) s'étalent sur un ordre de grandeur entre 5,7 (Harley et Pasternack, 1972) et 71 (Haque et Collinson, 1967) nGy par Bq h m ${ }^{-3}$ EEC. Des études détaillées de la contribution des différentes sources d'incertitude dosimétrique ont été effectuées par Birchall et James (1994), Porstendörfer (2001), Marsh et al. (2002), James et al. (2004) et Winkler-Heil et al. (2007).

Dans ces conditions, la similarité observée des facteurs de conversion, calculés selon des approches très différentes, est donc tout à fait remarquable. Elle met en lumière la pertinence des modèles établis et des hypothèses retenues et démontre l'applicabilité des outils mis en place. Ceci n'empêche bien sûr pas l'émergence de nouvelles études visant à préciser les coefficients obtenus et réduire les incertitudes associées. Dans ce domaine, le travail en cours du groupe de la CIPR sur la dosimétrie des émetteurs alpha devrait permettre de progresser encore et d'affiner les coefficients utilisés.

Enfin, la recherche permanente d'une meilleure précision dans les modèles dosimétriques ne doit pas faire oublier que l'un des concepts essentiels de la radioprotection réside dans la réduction des expositions autant que raisonnablement possible. Il devrait entrainer, dans le cas du radon, non pas des débats sans fin sur les valeurs des coefficients à utiliser mais la mise en place de mesures simples et facilement applicables, comme une ventilation adaptée des habitations, dont l'utilité n'est pas encore suffisamment enseignée, ni en France, ni dans le reste du monde.

\section{RÉFÉRENCES}

Birchall A., James A.C. (1994) Uncertainty analysis of the effective dose per unit exposure from radon progeny and implications for ICRP risk-weighting factors, Radiat. Prot. Dosim. 53(1-4), 133140 .

CIRC (1988) International Agency for Research on Cancer. Monographs on the Evaluation of Carcinogenic Risk to Humans: Manmade Fibres and Radon. Lyon: International Agency for Research on Cancer, IARC vol. 43.

Darby S., Hill D., Deo H., Auvinen A., Barros-Dios J.M., Baysson H., Bochicchio F., Falk R., Farchi S., Figueiras A., Hakama M., Heid I., Hunter N., Kreienbrock L., Kreuzer M., Lagarde F., Mäkeläinen I., Muirhead C., Oberaigner W., Pershagen G., Ruosteenoja E., Rosario A.S., 


\section{DOSIMÉTRIE DU RADON}

Tirmarche M., Tomásek L., Whitley E., Wichmann H.E., Doll R. (2006) Residential radon and lung cancer - detailed results of a collaborative analysis of individual data on 7148 persons with lung cancer and 14,208 persons without lung cancer from 13 epidemiological studies in Europe, Scand. J. Work Environ. Health 32(Suppl. 1), 1-83.

Haque A.K.M.M., Collinson AJ.L. (1967) Radiation dose to the respiratory system due to radon and its daughter products, Health Phys. 13, 431-443.

Harley N.H., Pasternack B.S. (1972) Alpha absorption measurements applied to lung dose from radon daughters, Health Phys. 23, 771-782.

ICRP Publication 30, Part 1 (1979) Limits for intakes of radionuclides by workers, Ann. ICRP 2(3/4).

ICRP Publication 32 (1981) Limits for inhalation of radon daughters by workers, Ann. ICRP 6(1).

ICRP Publication 60 (1991) 1990 Recommendations of the International Commission on Radiological Protection, Ann. ICRP 21(1-3).

ICRP Publication 65 (1993) Protection against radon-222 at home and at work, Ann. ICRP 23(2).

ICRP Publication 66 (1994) Human respiratory tract model for radiological protection, Ann. ICRP 24(1-3).

ICRP Publication 103 (2007) The 2007 recommendations of the International Commission on Radiological Protection, Ann. ICRP 37(2-4).

James A.C., Birchall A., Akabani G. (2004) Comparative dosimetry of BEIR VI revisited, Radiat. Prot. Dosim. 108(1), 3-26.

Krewski D., Lubin J.H., Zielinski J.M., Alavanja M., Catalan V.S., Field R.W., Klotz J.B., Létourneau E.G., Lynch C.F., Lyon J.L., Sandler D.P., Schoenberg J.B., Steck D.J., Stolwijk J.A., Weinberg C., Wilcox H.B. (2006) A combined analysis of North American case-control studies of residential radon and lung cancer, J. Toxicol. Environ. Health A 69(7), 533-597.

Lubin J.H., Wang Z.Y., Boice J.D.Jr., Xu Z.Y., Blot W.J., De Wang L., Kleinerman R.A. (2004) Risk of lung cancer and residential radon in China : pooled results of two studies, Int. J. Cancer 109(1), 132-137.

Marsh J.W., Birchall A., Butterweck G., Dorrian M.-D., Huet C., Ortega X., Reineking A., Tymen G., Schuler Ch., Vargas A., Vezzu G., Wendt J. (2002) Uncertainty analysis of the weighted equivalent lung dose per unit exposure to radon progeny in the home, Radiat. Prot. Dosim. 102(3), 229-248.

NEA (1983) Nuclear Energy Agency, Dosimetry aspects of exposure to radon and thoron and decay products. NEA expert report. OECD, Paris.

NRC (1999) National Research Council. Committee on Health Risks of Exposure to Radon. Board on Radiation Effects Research. Health effects of exposure to radon. Washington, D.C.: National Academy Press, USA. BEIR VI report.

Porstendörfer J. (2001) Physical parameters and dose factors of the radon and thoron decay products, Radiat. Prot. Dosim. 94(4), 365-373.

UNSCEAR (1982) United Nations Scientific Committee on the Effects of Atomic Radiation, Sources and effects of ionizing radiation. Report to the general assembly with annexes, United Nations, New York

UNSCEAR (1988) United Nations Scientific Committee on the Effects of Atomic Radiation, Sources, effects and risks of ionizing radiation. Report to the general assembly with annexes, United Nations, New York.

UNSCEAR (1993) United Nations Scientific Committee on the Effects of Atomic Radiation, Sources and effects of ionizing radiation. Report to the general assembly with scientific annexes, United Nations, New York. 
UNSCEAR (2000) United Nations Scientific Committee on the Effects of Atomic Radiation, Sources and effects of ionizing radiation. Report to the general assembly with scientific annexes, United Nations, New York.

UNSCEAR (2008) United Nations Scientific Committee on the Effects of Atomic Radiation, Sources and effects of ionizing radiation. Report to the general assembly with scientific annexes. United Nations, New York.

WHO (2007) World Health Organization international radon project, Report of the 3rd meeting, http://www.who.int.

Winkler-Heil R., Hofmann W., Marsh J.W., Birchall A. (2007) Comparison of radon lung dosimetry models for the estimation of dose uncertainties. Radiat. Prot. Dosim. 127(1-4), 27-30. 